\title{
Identification of the first homozygous 1-bp deletion in GDF9 gene leading to primary ovarian insufficiency by using targeted massively parallel sequencing
}

Monica M. França ${ }^{1}$, Mariana F. A. Funari ${ }^{1}$, Mirian Y. Nishi ${ }^{1,3}$, Amanda M. Narcizo $^{3}$, Sorahia Domenice ${ }^{1}$, Elaine M. F. Costa ${ }^{1}$, Antonio M. Lerario ${ }^{2,3}$, Berenice B. Mendonca ${ }^{1,3}$

${ }^{1}$ Unidade de Endocrinologia do Desenvolvimento, Laboratório de Hormônios e Genética Molecular/LIM42, Hospital das Clínicas, Disciplina de Endocrinologia, Faculdade de Medicina da Universidade de São Paulo, São Paulo, Brazil

${ }^{2}$ Department of Internal Medicine, Division of Metabolism, Endocrinology and Diabetes, University of Michigan, Ann Arbor, MI 48109, USA

${ }^{3}$ Laboratório de Sequenciamento em Larga Escala (SELA), Faculdade de Medicina da Universidade de São Paulo, São Paulo, Brazil

Corresponding Author: Monica Malheiros França

Hospital das Clínicas, Laboratório de Hormônios e Genética Molecular

Av. Dr. Enéas de Carvalho Aguiar, 155, $2^{\circ}$ andar, Bloco 6

CEP: 05403-900, São Paulo, Brasil.

Email: monicamalheiros@usp.br; beremen@usp.br

This is the author manuscript accepted for publication and has undergone full peer review but has not been through the copyediting, typesetting, pagination and proofreading process, which may lead to differences between this version and the Version of Record. Please cite this article as doi: $10.1111 /$ cge.13156

This article is protected by copyright. All rights reserved. 
Tel: 5511 2661-7512; Fax: 5511 2661-7519

\title{
Conflict of interest
}

The authors declare that they have no conflict of interest.

\begin{abstract}
Targeted massively parallel sequencing (TMPS) has been used in genetic diagnosis for Mendelian disorders. In the past few years, the TMPS has identified new and already described genes associated with primary ovarian insufficiency phenotype. Here, we performed a targeted gene sequencing to find a genetic diagnosis in idiopathic cases of Brazilian POI cohort. A custom SureSelect ${ }^{\mathrm{XT}}$ DNA target enrichment panel was designed and the sequencing was performed on Illumina NextSeq sequencer. We identified one homozygous 1-bp deletion variant (c.783delC) in the GDF9 gene in one patient with POI. The variant was confirmed and segregated using Sanger sequencing. The c.783delC GDF9 variant changed an aminoacid creating a premature termination codon (p.Ser262Hisfs*2). This variant was not present in all public databases (ExAC/gnomAD, NHLBI/EVS and 1000 Genomes). Moreover, it was absent in 400 alleles from fertile Brazilian women screened by Sanger sequencing. The patient's mother and her unaffected sister carried the c.783delC variant in a heterozygous state, as expected for an autosomal recessive inheritance. Here, the targeted massively parallel sequencing identified the first homozygous 1-bp deletion variant in GDF9. This finding
\end{abstract}


reveals a novel inheritance pattern of pathogenic variant in GDF9 associated with POI, thus improving the genetic diagnosis of this disorder.

Keywords: GDF9, Primary ovarian insufficiency, Targeted massively parallel sequencing, female infertility

\section{Introduction}

Primary ovarian insufficiency (POI), also known as premature ovarian failure (POF), results in amenorrhea, hypoestrogenism, infertility, and elevated gonadotropin levels $(1,2)$. The impairment of ovarian function could be induced by several factors, such as $\mathrm{X}$ chromosome abnormalities, genetic and environmental conditions. Several genes known to be involved in the recruitment, development, and maturation of the follicles and their constituent oocytes have been implicated in POI (2). Bone morphogenic proteins (BMPs), growth differentiation factors (GDFs), and neurotrophic factors (NGF, BDNF, and GDNF) are few types of growth factors associated with the follicular development pathway (2).

Targeted massively parallel sequencing has been shown to be a powerful tool to elucidate the genetic origin of the POI phenotype. Here, we describe the first homozygous 1-bp deletion in the GDF9 gene revealed by targeted gene 
massively parallel sequencing as a cause of primary ovarian insufficiency in one woman born to consanguineous parents.

\section{Patients and methods}

\section{Case report}

Approval from the institutional review board and written informed consent from all subjects were obtained before blood collection for DNA analysis. This study was approved by the Ethics Committee of Hospital das Clínicas, University of São Paulo Medical School, Brazil (protocol number 2015/12837/1.015.223).

From a cohort of 48 sporadic $\mathrm{POI}$ cases, the proband was selected for molecular analysis, following clinical criteria such as high levels of gonadotropins (FSH>40 U/L), hypoestrogenism, absence of spontaneous menarche and breast development, absence of FMR1 premutation, and autoantibodies. At first appointment, a 19-year-old woman had normal height and low weight $(151 \mathrm{~cm}$ and $32 \mathrm{Kg}$ ). Breast development and pubic hair were at Tanner II and III, respectively. The karyotype was $46, \mathrm{XX}$, as determined by the analysis of 50 metaphases. Basal serum gonadotropin levels were elevated in the proband $(\mathrm{FSH}=87 \mathrm{U} / \mathrm{L}$ and $\mathrm{LH}=51 \mathrm{U} / \mathrm{L})$, whereas serum estradiol was low (<13 pg/ml). 
The patient was treated with conjugated estrogens daily followed by progesterone replacement in the first 12 days of the month, resulting in complete breast development and normal menstrual cycle.

\section{Molecular Analyses}

Genomic DNA was extracted from peripheral blood leukocytes using standard procedures. A custom SureSelect ${ }^{\mathrm{XT}}$ DNA target enrichment panel was designed using SureDesign tools (Agilent Technologies Santa Clara, CA, USA). Based on female and male gonadal development and function, 284 known and candidate genes were selected. All exons and 25 base pairs of intronic flanking sequence were included. The proband's library was prepared according to the SureSelect XT Target Enrichment Protocol (Agilent Technologies Santa Clara, CA, USA). Sequencing was performed on a NextSeq 500 next-generation sequencer (Illumina San Diego, CA, USA). Alignment of raw data and variant calling were performed following the steps described by França and collaborators (3). The allelic variants identified were classified according to the American College of Medical Genetics and Genomics and the Association for Molecular Pathology (ACMG/AMP) (4).

The selected variant was confirmed by Sanger sequencing in the patient and her family. Primers flanking the GDF9 mutation (exon3:c.783delC) (ENSG00000164404/NM_005260.5) were used for PCR amplification. All PCR products were sequenced using BigDye terminator v3.1 cycle sequencing kit 
followed by automated sequencing using the ABI PRISM 3130xl (Applied Biosystems, Foster City, CA). Moreover, Sanger sequencing was performed to evaluate 200 fertile Brazilian women controls for the putative damaging variant found in this patient.

\section{Results}

Following filter criteria, we included only coding or splicing variants and variants expected by in silico prediction to be disease-causing, which were absent or present with minor allele frequency lower than $0.1 \%$ in the population databases (1000Genomes, ExAC, NHLBI/EVS). The targeted sequencing panel was able to identify one homozygous GDF9 variant in the proband. The c.783delC GDF9 variant is located in exon 3; it changes a serine to histidine at the position 262 and creates a premature termination codon (NM_005260.5 (GDF9_i001):c.783delC:p.Ser262Hisfs ${ }^{\star} 2$ ), as predicted by the Mutalyzer analysis. The mean coverage depth of the targeted regions in panel sequencing data was $145 \mathrm{x}(\mathrm{SD} \pm 66 \mathrm{x})$, with at least $99.38 \%$ of the sequenced bases covered more than 10 -fold. Moreover, the number of total sequenced reads in the proband was $1,610,860$ of which $99.72 \%$ corresponded to mapped reads. A total of 888 variants were called before filtering, of which eight variants were indels and 880 variants were SNVs.

Sanger sequencing confirmed the c.783delC GDF9 variant in a homozygous state in the patient and in a heterozygous state in her mother and her fertile 
sister. This variant was absent from all public databases [Genome and Exome Aggregation Consortium (ExAC/gnomAD), in the NHLBI Exome Sequencing Project (ESP) Exome Variant Server (EVS) and the Internacional Genome Sample Resource-1000 Genomes Project]. Moreover, the variant was not present in 400 alleles from fertile Brazilian women and in 609 elderly Brazilians exomes from ABraOM (Online Archive of Brazilian Mutations) database (5).

\section{Discussion}

The TGF- $\beta$ superfamily contains more than 35 members in vertebrates, including TGF- $\beta$ s, BMPs (bone morphogenetic proteins), GDFs (growth differentiation factors), activins, inhibins, and mullerian inhibiting hormone (6).

Some members of this family, such as GDF9 and BMP15, play different functions in the ovary by regulating somatic cell function and female fertility (6). As visualized by in situ hybridization analysis, GDF9 and BMP15 mRNAs are expressed specifically in the mouse oocyte of the preantral follicle type, but not in the primordial follicle. After these stages, expression of both persists in the oocyte during folliculogenesis and in ovulated oocytes (7), suggesting a functional relevance of these factors in ovary development.

In mice, the Gdf9 null model displayed the role of GDF9 in ovarian function. Gdf9-null female mice were infertile due to a block at the primary follicle stage (8). Their ovaries showed a block in growth and proliferation of granulosa cells, lack of theca layer development and oocyte defects, which included oocyte 
meiotic competence abnormalities and larger than normal growth of the oocyte $(8,9)$. In contrast, Gdf9-null male mice are fertile, in addition to heterozygous $\mathrm{Gdf9}^{+/-}$females (8).

Furthermore, sheep have also proved a valuable model to elucidate the importance of GDF9 in female reproductive function (10). In Belclare and Cambridge sheep, GDF9 ${ }^{577 F}$ heterozygous mutations are associated with increased ovulation rate, resulting in larger litter size than normal whereas homozygous carriers, in both breeds, are infertile (11).

In humans, all the GDF9 mutations described so far in different POI cohorts are heterozygous missense variants (12). The mutational screening of GDF9 in 127 Indian women with $\mathrm{POI}$ reported two rare heterozygous missense mutations (c.199A $>$ C and c.646G $>A$ ) in six patients of the cohort. Both missense mutations are localized in propeptide region of GDF9 protein, and was identified in women with secondary amenorrhea (13). In 2006, Veitia's group have identified one GDF9 heterozygous transversion c.557C >A in the proregion, leading to the change p.Ser186Tyr, in one patient with secondary amenorrhea from a European cohort of 203 women with POI (14). GDF9 mutation was also found in a United States cohort as a rare explanation for POI. Among 61 subjects, the majority of them Caucasian, one heterozygous missense variant (c.307C>T) in a highly conserved proprotein region was identified (p.Pro103Ser) (15). In contrast with previous described heterozygous missense mutations, we report the first homozygous 1-bp deletion (c.783delC) in GDF9 
gene in one Brazilian patient with primary amenorrhea, a more severe phenotype. This variant followed the guideline of The American College of Medical Genetics and Genomics (ACMG) to be considered pathogenic: it was absent from all international databases (1000Genomes, NHLBI/EVS, ExAC and gnomAD) and Brazilian controls; it segregated with disease in affected members, and was absent in unaffected family members; it occurred in a gene definitively known to cause the disease, and was a null variant in a gene where loss-of-function is a known mechanism of disease (4).

As previously shown, all heterozygous missense mutations in the GDF9 gene were reported in patients with secondary amenorrhea $(13,14,15)$ suggesting a correlation between a more severe phenotype, as primary amenorrhea, and the loss-of-function variant. Moreover, consistent with Mendelian expectations for an autosomal-recessive mode of inheritance, the patient's mother, whom had normal ovarian function and had four children, was heterozygous for the same mutation demonstrating that some heterozygous GDF9 variants would not induce POI. Thus, we can suggest that POI phenotype induced by GDF9 mutations may have an autosomal dominant or recessive inheritance pattern.

According to Prosite_SIB database of protein domain, the TGF-beta domain region in GDF9 is localized between 316 and 454 amino acids. Furthermore, mutations in the proregion can affect the processing of proregion cleavage and could compromise normal growth and maturation of ovarian cells. Interesting, all described mutations were localized in the propeptide region of GDF9 as well as 
the variant reported here (p.Ser262Hisfs*2), and therefore could result in a truncated protein missing a TGF-beta domain.

In conclusion, we report the first homozygous 1-bp deletion in GDF9. This finding reveals a novel inheritance pattern of a pathogenic variant in GDF9 associated with POI, thus improving the genetic diagnosis of this disorder.

\section{Acknowledgments}

The authors wish to thank the patients and their families for participating in this study. They are grateful to LIM/42 and SELA teams for all technical assistance. This work was supported by the Fundação de Amparo à Pesquisa do Estado de São Paulo Grant 2014/14231-0 (to M.M.F.); Fundação de Amparo à Pesquisa do Estado de São Paulo Grant 2013/02162-8, Nucleo de Estudos e Terapia Celular e Molecular (NETCEM) and Conselho Nacional de Desenvolvimento Científico e Tecnológico Grant 303002/2016-6 (to B.B.M.); Fundação de Amparo à Pesquisa do Estado de São Paulo Grant 2014/50137-5 (to SELA)

\section{References}

1. Shelling AN. Premature ovarian failure. Reproduction review. 2010; 140: 633-641. 
2. Tucker EJ, Grover SR, Bachelot A, Touraine P, et al. Premature ovarian insufficiency: New perspectives on genetic cause and phenotypic spectrum. Endocr Rev. 2016; 37(6):609-635.

3. França MM, Lerario AM, Funari MFA, et al. A novel homozygous missense FSHR variant associated with hypergonadotropic hypogonadism in two siblings from a Brazilian family. Sex Dev. 2017; 11(3):137-142.

4. Richards S, Aziz N, Bale S, et al. ACMG Laboratory Quality Assurance Committee: Standards and guidelines for the interpretation of sequence variants: a joint consensus recommendation of the American College of Medical Genetics and Genomics and the Association for Molecular Pathology. Genet Med. 2015; 17(5):405-424.

5. Naslavsky MS, Yamamoto GL, de Almeida TF, et al. Exomic variants of na elderly cohort of Brazilians in the ABraOM database. Hum Mutat. $2017 ; 38(7): 751-763$.

6. Chang $\mathrm{H}$, Brown $\mathrm{CW}$, Matzuk MM. Genetic analysis of the mammalian TGF- $\beta$ superfamily. Endocr Rev. 2002; 23:787-823.

7. Elvin JA, Yan C, Matzuk MM. Oocyte-expressed TGF-beta superfamily members in female fertility. Mol Cell Endocrinol. 2000; 159(1-2):1-5.

8. Dong J, Albertini DF, Nishimori K, et al. Growth differentiation factor-9 is required during early ovarian folliculogenesis. Nature. 1996; 383(6600):531-535. 
9. Carabatsos MJ, Elvin J, Matzuk MM, et al. Characterization of oocyte and follicle development in growth differentiation factor-9-deficient mice. Dev Biol. 1998; 204(2):373-384.

10. Otsuka Fm McTavish KJ, Shimasaki S. Integral role of GDF-9 and BMP15 in ovarian function. Mol Reprod Dev. 2011; 78(1):9-21.

11. Hanrahan JP, Gregan SM, Mulsant $P$, et al. Mutations in the genes for oocyte derived growth factors GDF9 and BMP15 are associated with both increased ovulation rate and sterility in Cambridge and Belclare sheep (Ovis aries). Biology of Reproduction. 2004; 70:900-909.

12. Rossetti R, Ferrari I, Bonomi M, et al. Genetics of primary ovarian insufficiency. Clin Genet. 2017; 91(2):183-198.

13. Dixit $\mathrm{H}$, Rao LK, Padmalatha $\mathrm{V}$, et al. Mutational screening of the coding region of growth differentiation fator 9 gene in Indian women with ovarian failure. Menopause. 2005; 12:749-754.

14. Laissue $P$, Christian-Maitre $S$, Touraine $P$, et al. Mutations and sequence variants in GDF9 and BMP15 in patients with premature ovarian failure. Eur J Endocrinol. 2006; 154(5):739-744.

15. Kovanci E, Rohozinski J, Simpson JL, et al. Growth differentiating factor9 mutations may be associated with premature ovarian failure. Fertil Steril. 2007; 87(1):143-146. 


\section{Legend}

Figure 1: The GDF9 homozygous pathogenic variant c.783delC found in the proband. (A) Family Pedigree. The black arrow indicates the proband (II-1). Pedigree numbers of individuals are indicated above the symbols. (B) Screenshots from the Integrative Genomics Viewer of targeted sequencing data from the proband (II-1). On top, the [hg19] genomic coordinates shown on the screen. In the middle section, gray bars represent sequencing reads with a histogram of individual base coverage above the reads. The red arrow indicates the homozygous deletion, which is clearly visible in the middle of the panel as a region of decreased sequencing coverage. (C) The electropherogram confirmed homozygous c.783delC (red arrow on nucleotide peak of interest) in II-1 and heterozygous in mother and sister (I-2 and II-3). WT denotes the wild-type allele and MT indicates the c.783delC variant. An asterisk indicates samples utilized for targeted massively parallel sequencing (TMPS); POI: primary ovarian insufficiency. 

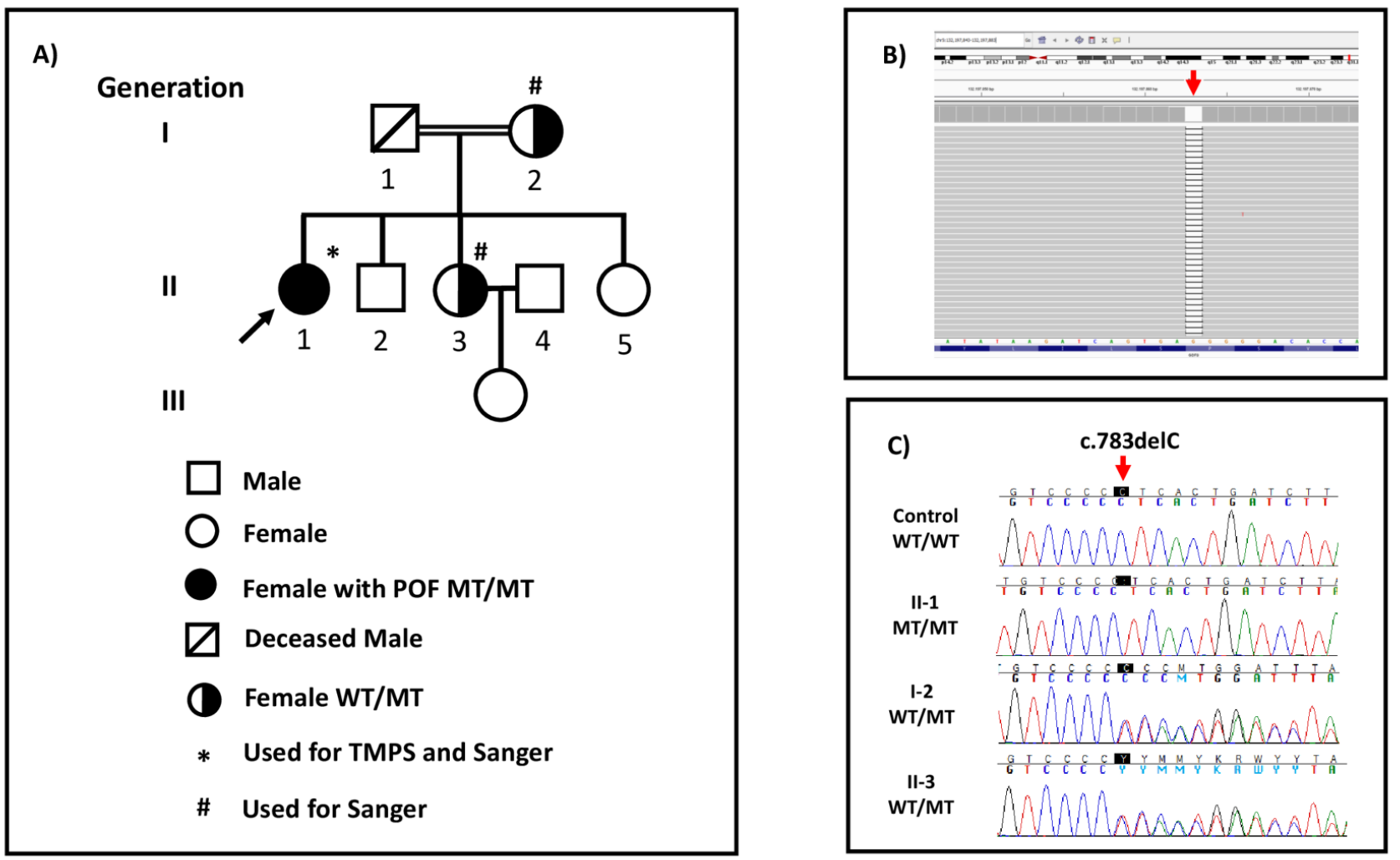

Figure 1

Franca-etal_GDF9_Figure-1_A_B_C_final_edit_review.tiff 


\section{TMPS identified the first homozygous c.783delC in GDF9 gene in one patient with POF}

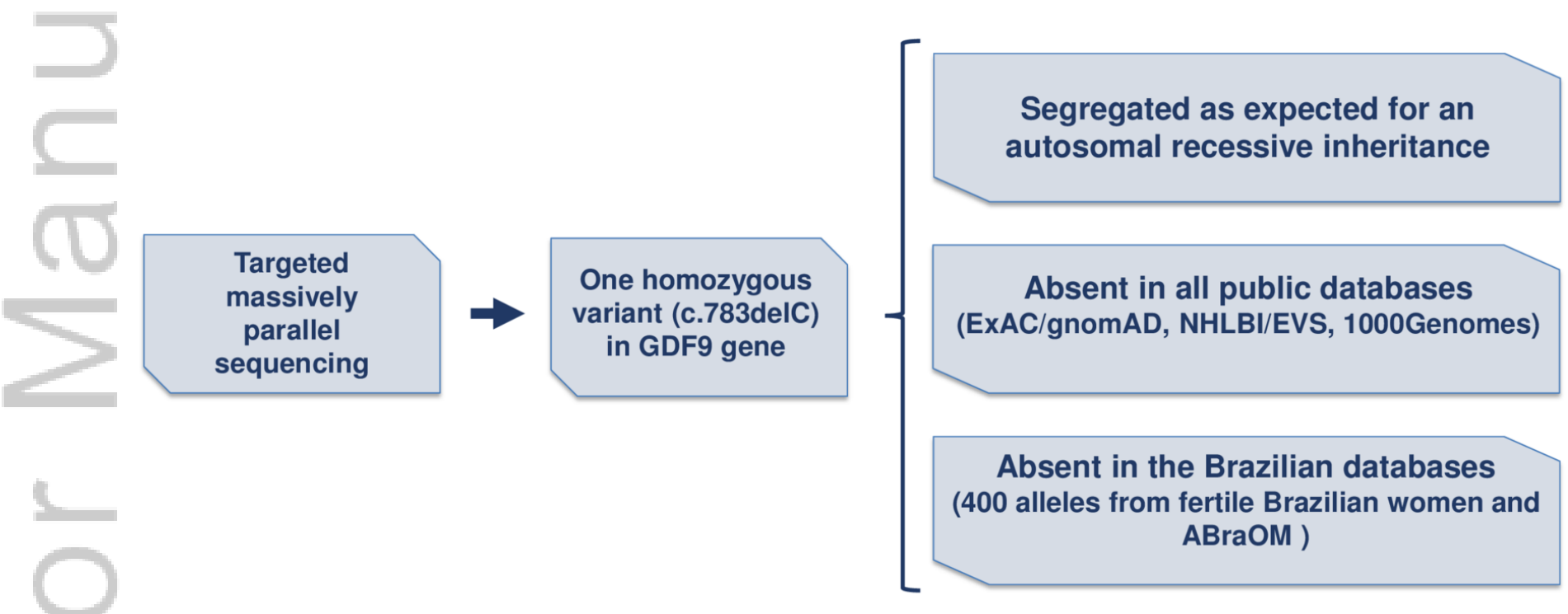

Franca-etal_Grafical-abstract_out2017.tiff

This article is protected by copyright. All rights reserved. 